\title{
The role of juvenile hormone in regulating reproductive physiology and dominance in Dinoponera quadriceps ants
}

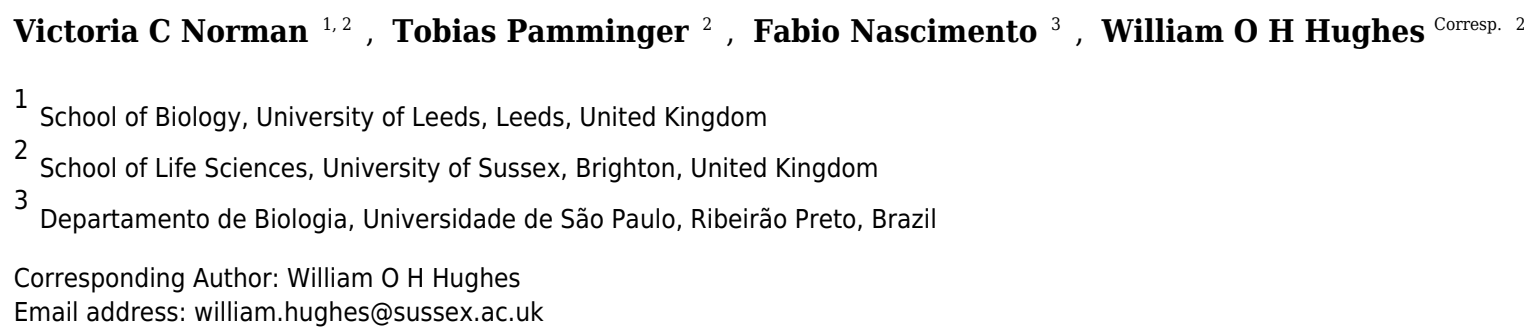

Unequal reproductive output among members of the same sex (reproductive skew) is a common phenomenon in a wide range of communally breeding animals. In such species, reproductive dominance is often acquired during antagonistic interactions between group members that establish a reproductive hierarchy in which only a few individuals reproduce. Rank-specific syndromes of behavioural and physiological traits characterize such hierarchies, but how antagonistic behavioural interactions translate into stable rankspecific syndromes remains poorly understood. The pleiotropic nature of hormones makes them prime candidates for generating such syndromes as they physiologically integrate environmental (social) information, and often affect reproduction and behaviour simultaneously. Juvenile hormone $(\mathrm{JH})$ is one of several hormones that occupy such a central regulatory role in insects and has been suggested to regulate reproductive hierarchies in a wide range of social insects including ants. Here we use experimental manipulation to investigate the effect of $\mathrm{JH}$ levels on reproductive physiology and social dominance in high-ranked workers of the eusocial ant Dinoponera quadriceps, a species that has secondarily reverted to queenless, simple societies. We show that JH regulated reproductive physiology, with ants in which JH levels were experimentally elevated having more regressed ovaries. In contrast, we found no evidence of JH levels affecting dominance in social interactions. This could indicate that $\mathrm{JH}$ and ovary development are decoupled from dominance in this species, however only high-ranked workers were investigated. The results therefore confirm that the regulatory role of $\mathrm{JH}$ in reproductive physiology in this ant species is in keeping with its highly eusocial ancestors rather than its secondary reversion to simple societies, but more investigation is needed to disentangle the relationships between hormones, behaviour and hierarchies. 
1 The role of juvenile hormone in regulating reproductive

2 physiology and dominance in Dinoponera quadriceps ants

4 Victoria C. Norman ${ }^{1,2}$, Tobias Pamminger ${ }^{2}$, Fabio Nascimento ${ }^{3}$ \& William O. H.

5 Hughes $^{2}$

6

7

$8{ }^{1}$ School of Biology, University of Leeds, Leeds, United Kingdom

$9 \quad{ }^{2}$ School of Life Sciences, University of Sussex, Brighton, United Kingdom

10 32Departamento de Biologia, Universidade de São Paulo, Ribeirão Preto, Brazil

11

12

13

14 Corresponding author William Hughes; william.hughes@sussex.ac.uk 


\section{ABSTRACT}

17 Unequal reproductive output among members of the same sex (reproductive skew) is a common phenomenon in a wide range of communally breeding animals. In such species, reproductive dominance is often acquired during antagonistic interactions between group members that establish a reproductive hierarchy in which only a few individuals reproduce. Rank-specific syndromes of behavioural and physiological traits characterize such hierarchies, but how antagonistic behavioural interactions translate into stable rank-specific syndromes remains poorly understood. The pleiotropic nature of hormones makes them prime candidates for generating such syndromes as they physiologically integrate environmental (social) information, and often affect reproduction and behaviour simultaneously. Juvenile hormone $(\mathrm{JH})$ is one of several hormones that occupy such a central regulatory role in insects and has been suggested to regulate reproductive hierarchies in a wide range of social insects including ants. Here we use experimental manipulation to investigate the effect of $\mathrm{JH}$ levels on reproductive physiology and social dominance in high-ranked workers of the eusocial ant Dinoponera quadriceps, a species that has secondarily reverted to queenless, simple societies. We show that JH regulated reproductive physiology, with ants in which $\mathrm{JH}$ levels were experimentally elevated having more regressed ovaries. In contrast, we found no evidence of JH levels affecting dominance in social interactions. This could indicate that $\mathrm{JH}$ and ovary development are decoupled from dominance in this species, however only high-ranked workers were investigated. The results therefore confirm that the regulatory role of $\mathrm{JH}$ in reproductive physiology in this ant species is in keeping with its highly eusocial ancestors rather than its secondary reversion to simple societies, but more investigation is needed to disentangle the relationships between hormones, behaviour and hierarchies. 


\section{INTRODUCTION}

40 In many group-living animals, reproduction is not equally distributed among the breeding

41 members. This phenomenon, known as reproductive skew, occurs in a wide range of communally-

42 breeding species including birds, fishes and insects (Jamieson 1997; Cuvillier-Hot et al. 2004a;

43 Neff et al. 2008). Due to its fundamental implications for both ecological and evolutionary

44 processes, this topic has attracted attention over the past decades from both a theoretical (see

45 Kokko and Johnstone 1999; Johnstone 2000; Kokko 2003) and empirical perspective in a variety

46 of study systems (Bourke et al. 1997; Field et al. 1998; Reeve and Keller 2001; Widdig et al.

47 2004).

48 Social insects, in particular ants, have emerged as important model systems to test some

of the main predictions of reproductive skew theory due to their wide range of social complexity and life history strategies (Reeve and Keller 2001). The majority of ant species are highly eusocial, with complex societies in which a one or several queens produce all female offspring and her unmated daughter workers perform all other tasks such as brood care, foraging and nest defence (Hölldobler and Wilson 1990). In such systems, as a result of the haplodiploid sex determination in Hymenoptera, workers are only able to produce male offspring and conflicts between queens and workers, or between workers, arise over male parentage (Ratnieks and Reeve 1992; Ratnieks et al. 2006). This group conflict and how it is resolved has generated a

57 plethora of ground-breaking work, revolutionizing our understanding of group formation, conflict and maintenance (Ratnieks et al., 2006).

While most modern ants have a specialized queen caste, some genera, such as Dinoponera, have secondarily reverted to simple, queenless societies in which reproduction is monopolized instead by mated, reproductively active workers called gamergates (Peeters 1997). 
62 In most gamergate systems, all workers have the potential to become the dominant reproductive,

63 resulting in strong within-group conflict over reproduction (Peeters 1997). Such conflicts are

64 often resolved via aggressive behavioural interactions that establish a dominance hierarchy in

65 which only a single, or a small group of workers go on to reproduce. The question of how such

66 ritualized physical aggression is physiologically translated into stable reproductive hierarchies

67 with lower ranked workers not only remaining reproductively inactive but also assuming helper

68 roles, remains poorly understood. In some species such as D.quadriceps, subordinate workers

69 play a role in stabilizing the dominance hierarchy (Monnin and Peeters 1999).

70 Hormones are prime candidates for the proximate mechanisms underlying this process,

71 because they not only physiologically integrate social stimuli including stress, but also regulate

72 numerous other essential processes in adult insects such as reproduction, maternal behaviour and

73 aggression (Nijhout 1998; Sasaki et al. 2007; Tibbetts and Huang 2010). There are several

74 hormones that have been implicated in this, including dopamine, ecdysone and vitellogenin, with

75 juvenile hormone $(\mathrm{JH})$ being perhaps the best studied. However, it appears that $\mathrm{JH}$ can have

76 contrasting effects in different taxa. In primitively eusocial species, such as paper wasps and

77 bumblebees, $\mathrm{JH}$ is gonadotropic, stimulating ovary development in the same way as in solitary

78 insects and resulting in individuals being more socially dominant, while in the highly eusocial

79 honeybee, in contrast, JH has lost its gonadotropic effect and instead is involved in regulating

80 division of labour (Robinson and Vargo 1997). In highly eusocial Lasius niger ants, the

81 gonadotropic effect of JH has not only been lost but reversed, with higher JH levels being

82 associated with reduced egg production (Pamminger et al. 2016a, b). In addition JH has been

83 shown to trigger foraging behaviour in some ants, making it a possible candidate to coordinate not

84 only reproductive division of labour, but also division of labour between workers (Robinson and 
85

86

87

88

89

90

91

92

93

94

95

96

97

Vargo 1997; Norman and Hughes 2016). This might suggest a relatively simple switch in the action of $\mathrm{JH}$ with the evolution of complex eusocial societies, but it appears that the evolution dynamics of JH mode of action is more complex than that. In Solenopsis and Pogonomyrmex, ant genera with complex societies, JH exhibits stimulatory functions during reproduction (Brent and Vargo 2003; Libbrecht et al. 2013). In Streblognathus and Diacamma, ants with simple, queenless societies, low $\mathrm{JH}$ titres in gamergates correlates with high individual ranks within the hierarchy and JH application will result in a loss of the reproductive status of the alpha (Sommer et al. 1993;

Cuvillier-Hot et al. 2004b; Brent et al. 2006). In Harpegnathos ants, which also have simple, societies, where gamergates can reproduce following the founding queen's death, JH levels do not differ between reproductive and non-reproductive individuals, and experimental elevation of $\mathrm{JH}$ levels through the application of Juvenile Hormone analogue had no effect on egg production (Penick et al. 2011).

Here we investigate the effects of $\mathrm{JH}$ on reproductive physiology and social dominance in the queenless ponerine ant Dinoponera quadriceps, by using topical application of the JH analogue $(\mathrm{JHa})$ methoprene to experimentally manipulate $\mathrm{JH}$ levels. This species is of particular interest because it is one of ca. 100 species to have undergone an evolutionary reversion from a highly eusocial ancestor with a queen caste back to its basal state with queenless, simple societies (Peeters 1997; Monnin and Peeters 1998). All females in D. quadriceps are morphologically identical, with a single dominant gamergate, the alpha, actively suppressing a group of the higher ranked workers from becoming reproductively active with ritualized physical aggression including antennal blocking and boxing (Monnin and Peeters 1998; Grainger et al. 2014). The presence of an alpha within the colony not only inhibits ovary activation in workers, the first step towards becoming reproductively active, but also results in submissive behaviour by 
108 subordinates (Smith et al. 2011; Asher et al. 2013). The physiological phenotypic differences

109 between alphas and subordinates result from subtle differences in transcriptional network

110 organisation, involving both conserved and novel genes (Patalano et al. 2015). If JH functions

111 similarly to other queenless ant species, then we predict JH will suppress ovarian development

112 and cause high ranking workers to decrease in status. If $\mathrm{JH}$ functions as a gonadotropin, similar

113 to solitary insects and social wasps, then we predict $\mathrm{JH}$ to activate ovarian development and

114 potentially to move up in the hierarchy. We focus for our experimental manipulation on high-

115 ranked, but not alpha, workers because these have both the potential to move up the hierarchy to

116 become reproductives and the potential to lose their position in the social hierarchy and become

117 middle or low-ranked workers. We measure the effect of JH manipulation on ovary development

118 and dominance behaviour. If JH links reproduction and hierarchy-related behaviours it would

119 then provide a proximate physiological explanation for rank-associated trait syndromes.

METHODS

123 We used 13 colonies of $D$. quadriceps, which were collected from Bahia state, Brazil in

124 November 2014 under permit from Instituto Brasileiro do Meio Ambiente e dos Recursos

125 Naturais (IBAMA; 14BR004553). All colonies were maintained in the lab at $27^{\circ} \mathrm{C}$ and $80 \%$

126 relative humidity for at least six months before the experiment. Colonies were fed with Tenebrio

127 molitor larvae and apple, and had ad libitum access to water. Each individual was uniquely

128 marked on the pronotum with numbered tags.

129

130 Establishing the dominance hierarchy 
131 Firstly, to establish the dominance hierarchy, colonies were monitored daily for two weeks, with

132 the behaviours and locations of each individual being recorded once each day for 14 days.

133 Observations lasted until each individual per colony had been recorded. Individuals showed high

134 levels of consistency in behaviour and location during this period. Given the positive association

135 in this species between an individual being of high rank and it interacting with brood (Monnin

136 and Peeters 1999; Asher et al. 2013), any individual that was observed at least once interacting

137 with brood over the 14 day initial observation period was selected to undergo pairwise isolated

138 dyadic interactions to narrow down their position in the social hierarchy. This method pairs

139 every combination of ants sampled to observe which individual in each dyad is the dominant and

140 which the subordinate, based on a characteristic dominance behaviour; this has previously been

141 shown to be a reliable and robust way to establish dominance hierarchies in this species

142 (Grainger et al. 2014). For this, individuals were taken from their colonies and placed

143 individually in pots ( $85 \mathrm{~mm} \times 75 \mathrm{~mm}$ x $55 \mathrm{~mm})$ and allowed to acclimatise for $15 \mathrm{~min}$. Pairs of

144 ants were then placed in a new pot, their dominance interaction was observed and the dominant

145 ant was recorded. This is indicated by only one behaviour in this context: dominant ants stand

146 tall with their antennae either side of the subordinate individual which has antennae laid flat back

147 behind their head (Grainger et al., 2014). This reaction normally occurs within the first $60 \mathrm{~s}$ of

148 contact between pairs when it is expressed. We then ranked individuals based on the number of

149 times they expressed dominance and assigned ranks to each individual. The higher-ranking

150 individuals that ranked directly below the alpha and clearly above the remainder of the colony

151 were then selected for the study (two or three high-ranking workers per colony, and 32 in total).

152 Of these, 16 high-ranking workers were treated with JHa (at least one per colony) and 16 as

153 controls (at least one per colony; see below). 


\section{Worker size and weight}

156 Before the start of the experiment all selected workers were immobilized on ice for $1 \mathrm{~min}$ and

157 their head width (maximal interorbital distance) measured as proxy for body size, as well as their 158 fresh weight using a Precisa $125 \mathrm{~A}$ balance.

\section{Behavioural measures and experimental procedure}

Behavioural observations were made daily for five days before the first application of treatments

to determine how consistent ants were for a number of behavioural variables. In total,

We carried out daily scans for five days prior to treatment in which we recorded for each focal ant whether or not it was showing any aggression (either within the nest to conspecifics or gaping it's mandibles in defence outside of the nest), whether or not it was showing any brood care behaviours, and two measures of 'sociability': the distance to the nearest ant and the number of ants within $5 \mathrm{~cm}$ of the focal ant ('contacts'). During the same time interval we carried out individual-level assays for activity level, 'boldness' and defensive aggression, with the expectation that high rankers would show low activity level (Monnin et al. 2003), low boldness

171 (as they are based inside and away from any 'risky' tasks such as nest defence or foraging

172 (Nascimento et al. 2012; Asher et al. 2013), and high levels of aggression (known to be associated with higher ranks (Monnin and Peeters 1999; Cant et al. 2006)). General activity level

174 was determined simply by placing the focal ant in a $90 \mathrm{~mm}$ Petri dish lined with filter paper,

175 leaving it to acclimatise for $2 \mathrm{~min}$, and then videoing the ant for 5 min using a Logitech c920

176 webcam. Speed of movement was quantified from videos using Antrak path analysis software 
177 (Tranter et al. 2014). 'Boldness' was determined by placing the focal ant in a $90 \mathrm{~mm}$ Petri dish

178 lined with filter paper and half blackened out with tape across the lid and sides, leaving it to

179 acclimatise for $2 \mathrm{~min}$, and then videoing the ant for $5 \mathrm{~min}$ to allow the proportion of time spent in

180 the light half of the Petri dish to be calculated (less bold ants spend more time hiding in the

181 darkened area of the Petri dish). Defensive aggression was determined by placing the focal ant in

182 a pot ( $85 \mathrm{~mm} \times 75 \mathrm{~mm} \times 55 \mathrm{~mm})$, leaving it to acclimatise for $5 \mathrm{~min}$, and then tapping it gently

183 on the head with the tip of a toothpick, as in Pamminger et al. (2014). The reaction of the ant was

$184 \operatorname{ranked}(0=$ ignore, $1=$ antennate, $2=$ gape mandibles in a threat response, $3=$ bite $)$.

Following the initial assessment of individual behaviour, ants were assigned randomly to

186

187

188

189

190

191

192

193

194

195

196

197

198

199

either the methoprene treatment or acetone solvent control (coA) (with at least 1 methoprene

treated and 1 control treated ant per colony), and all subsequent behavioural observations were

conducted with the observer blind to the treatment. For the methoprene treatment, a dose of 16.5

$\mu \mathrm{g}$ of methoprene $\left(\mathrm{PESTANAL}^{\circledR}{ }^{\text {Sigma }}\right.$ Adrich $^{\odot}$ ) in $5 \mu$ lacetone was applied to the pronotum

three times over a period of 1 week; control ants received $5 \mu$ lacetone on the same occasions.

This dose was determined during a preliminary experiment and is low compared to the amounts

used in other social insect studies (Table S1), indicating that the observed effects are not caused

by potential toxic effects of $\mathrm{JH}$ at high doses. After two days of acclimatisation post-treatment,

we repeated the behavioural observations. We carried out the assays daily for 4 days and on the

$5^{\text {th }}$ day carried out dyadic interaction assays between the focal ants and all other workers that had

been observed performing brood care behaviour over the past three weeks. This enabled us to

determine if the methoprene treatment had not only affected behaviour but also the position of

the focal high rank ants in the hierarchy. Following the dyadic interactions, ants were freeze-

killed in liquid nitrogen and stored at $-80{ }^{\circ} \mathrm{C}$ until ovary dissection.

PeerJ reviewing PDF | (2018:02:25767:1:1:NEW 9 Jan 2019) 
201 Ovary dissection and fertility estimates

202 Ant ovaries were dissected under a Leica S8AP0 stereo microscope and the ovaries were

203 transferred into Ringer solution. The ovaries were photographed using a Leica DFC 295 Camera

204 and the Leica application suite software v. 4.1.0. Three ovarioles were randomly selected for

205 further analysis to keep consistency between individuals. Using a Pyser-SGI ${ }^{\circledR}$ S78 stage

206 micrometer 1.0/0.01 $\mathrm{mm}$ and the software ImageJ $1.47 \mathrm{v}$, we measured the minimum, maximum

207 and average width of the third of the ovarioles most end closest to the oviduct (containing the

208 furthest developed eggs if present) and the number of vitellogenic eggs, which are the white

209 (yolk), non-transparent and non-deformed portion of the eggs found in the ovarioles.

210

211 Statistical analysis

212 For the fertility analysis we carried out individual wilcoxin-signed rank tests for each of the

213 measures of fertility (minimum, maximum and average ovariole width and number of

214 vitellogenic eggs) as response variables against treatment (either methoprene or acetone control $215(\operatorname{coA}))$.

For the behavioural statistical analysis, we used the programme PRIMER 6, version

217 6.1.13, + add-in, version 1.0.3 (PRIMER-E Ltd) to perform permutational multivariate analysis

218 of variance (PERMANOVA). PERMANOVA is a non-parametric MANOVA, which has the

219 advantage that it is free from assumptions on data distributions (Anderson et al. 2008). All tests

220 were carried out using 9,999 permutations on a resemblance matrix using Euclidian distance as a

221 distance estimate. In all cases we used treatment as a fixed factor and colony as a random

222 predictor variable to account for the structured nature of the data. Interaction between the factors 
223 was included, but removed from the final minimum adequate model when nonsignificant. All

224 response variables were z-transformed prior to treatment in order to account for difference in

225 units and variation between variables, which facilitates the interpretation of results in particular

226 interactions between variables (Gotelli and Ellison 2004).

227 To test for potential differences in weight and size between workers belonging to

228 different colonies and treatments, both were used as response variables in a PERMANOVA. We

229 also used PERMANOVA to investigate the effects of treatment and colony on fertility and

230 behaviour. We used the change in behaviour following JH treatment as response variables for

231 analysis. We calculated the mean behaviour and hierarchy position (number of winning,

232 dominant encounters) before and after treatment to obtain a robust estimate for brood care,

233 aggression, 'boldness', activity and sociability (ants in close proximity and distance to the

234 nearest ant) and position in the hierarchy before and after treatment. We then calculated the

235 change in behaviour in response to treatment by subtracting the averaged behaviour value before

236 treatment from the average value after treatment; positive values therefore indicate an increase

237 and negative values a decrease in response to treatment. The same calculation was performed for

238 the change in rank (number of encounters won in dyadic interactions). To further explore the

239 qualitative differences between the treatments, we performed a one-way similarity of percentage

240 (SIMPER) analysis, a data exploration technique that calculates the contributions individual

241 factors make to both group (treatment) coherence and separation in a multidimensional scaling

242 (MDS) analysis. 
246 The experimental ants did not differ in size or weight between treatments or colonies

247 (respectively: Pseudo $\mathrm{F}_{1,31}=0.35, \mathrm{P}=0.72$; Pseudo $\mathrm{F}_{11,31}=1.04, \mathrm{P}=0.4$; Table 1), and worker

248 fertility also did not differ between colonies (Pseudo $\left.\mathrm{F}_{11,31}=1.82, \mathrm{P}=0.11\right)$. However, worker

249 fertility was affected by treatment, with JHa-treated individuals being less fertile compared to

250 those treated with acetone control under all measures of fertility taken here (oocyte number,

251 average ovariole width, minimum ovariole width and maximum ovariole width; 22; Table 1;

252 Table 2; Figure 1). The CoA ants were more variable in fertility than the JHa group (Figure 1).

253 Furthermore, there were smaller differences in fertility within the JHa group (Figure 1). In

254 contrast to the effects of JHa treatment on worker fertility, we found no significant differences

255 between treatment or colonies on social dominance behaviour (respectively: Pseudo $\mathrm{F}_{1,31}=0.74$,

$256 \mathrm{P}=0.6 ;$ Pseudo $\mathrm{F}_{11,31}=1.37, \mathrm{P}=0.06 ;$ Figure 2).

257

258

259 DISCUSSION

260 Our results show that $\mathrm{JH}$ has a role in regulating reproduction in D. quadriceps. Experimentally

261 elevated JH levels not only decreased the number of vitellogenic eggs in high-ranked workers,

262 but also resulted in an overall decrease in the size of individual ovarioles, indicating a substantial

263 reduction in reproductive potential. In solitary insects $\mathrm{JH}$ often has the opposite effect by

264 stimulating the production of vitellogenic oocytes and the same is true for many primitively

265 eusocial insects, such as non-swarm founding wasps and bumblebees (Robinson and Vargo

266 1997). It has been suggested that the functional reversal of JH in reproduction was important in

267 the evolution of complex societies, and a large number of studies demonstrate often radical

268 changes in the regulatory architecture of reproduction in eusocial species (Robinson and Vargo 
269 1997; Hartfelder 2000; Bloch et al. 2009). The classic example for this argument is the

270 remodelling of the regulatory function of $\mathrm{JH}$ in honeybees and some ants (Robinson and Vargo

271 1997; Bloch et al. 2009; Pamminger et al. 2016a; Azevedo et al. 2016; Pamminger et al. 2016b),

272 however a small number of studies clearly indicates that high social organization is possible

273 without it (e.g. Brent and Vargo 2003; Kelstrup et al. 2015). D. quadriceps supports the former

274 findings by demonstrating that a remodelling of $\mathrm{JH}$ function which inhibits reproduction can also

275 be associated with simple social organization. This makes sense given the evolutionary position

276 of D. quadriceps, with Dinoponera having secondarily reverted to simple, queenless societies

277 from a highly eusocial ancestor (Peeters 1997; Monnin and Peeters 1998; Monnin and Peeters

278 1999). This further supports the notion that there is likely no causal link between the remodelling

279 of the $\mathrm{JH}$ function in reproduction and the organisational complexity of insect societies.

280

The results presented here, that JH significantly decreases fertility, are therefore

281

282

283

284

285

286

287

288

289

290

291

consistent with those for other gamergate-led ant societies (Harpegnathos, Streblognathus and

Diacamma; Sommer et al. 1993; Cuvillier-Hot et al. 2004b; Penick et al. 2011). The role of JH in

such societies is thus relatively clear, but there remain questions on how $\mathrm{JH}$ functions in true

queens of other species. Importantly in these societies $\mathrm{JH}$ appears to be involved as part of an

'honest' signal in inform other colony members about their fertility (Cuvillier-Hot et al. 2004b),

a key cue for the maintenance of dominance hierarchies in these societies. In studies on a

gamergate ant society, a social wasp and termite - topical applications of $\mathrm{JH}$ affect cuticular

hydrocarbon $(\mathrm{CHC})$ profiles of adult and larval individuals such that the profile becomes more

'reproductive-like' and is perceived so by colony members (Kelstrup et al. 2014; Brent et al.

2016; Penick and Liebig 2017). However in Streblognathus ants alphas treated with JH had their

fertility reduced and the $\mathrm{CHC}$ profile matched more closely with that of a sterile worker than an 
292 alpha (Cuvillier-Hot et al. 2004a)

293 In contrast to the relatively well-studied effects of $\mathrm{JH}$ on reproductive physiology, little is

294 known about the regulatory role of $\mathrm{JH}$ in behaviour for most insects. The association between $\mathrm{JH}$

295 and aggression, maternal behaviour and activity has been documented in insects (Nijhout 1998;

296 Pearce et al. 2001; Tibbetts and Izzo 2009; Tibbetts et al. 2013), but these studies are restricted to

297 only a handful of species. In the honey bee Apis mellifera, JH, in combination with the yolk pre-

298 cursor vitellogenin, regulates one of the major behavioural transitions in the adult honeybee

299 worker from within-nest behaviour to external foraging (Robinson and Vargo 1997). This

300 transition is associated with a major remodelling of the behaviour repertoire and indicates the far-

301 reaching regulatory potential of $\mathrm{JH}$ in behaviour. A similar function of $\mathrm{JH}$ has been documented

302 in Pogonomyrmex californicus harvester ants and Acromyrmex echinator leaf-cutting ants

303 (Dolezal et al. 2009; Norman and Hughes 2016), demonstrating that JH can generate forager-like

304 behavioural phenotypes. In contrast to our expectations, we find no measurable effects of JH on

305 worker behaviour or position in the hierarchy in D. quadriceps. This could indicate that $\mathrm{JH}$,

306 fertility and dominance are decoupled in D. quadriceps. Indeed, a lack of behavioural effect of

307 JHa on alphas in Streblognathus ponerine ants has been reported previously (Cuvillier-Hot et al.

308 2004a). However, it is more likely that our study simply lacked the power to detect an effect. Our

309 study was deliberately focussed on only high-ranked workers because these were the individuals

310 in which both positive and negative effects could potentially be seen, and it may be that inclusion

311 of ants from the full spectrum of the social hierarchy or using in-nest behavioural observations

312 may reveal effects. 


\section{CONCLUSIONS}

316 Although JH is now known to have an important (though variable) role in the physiology of

317 reproductive dominance in social insects, other hormones are also likely to be as, or more,

318 important to reproductive status and social dominance in D. quadriceps. In particular, ecdysone

319 or vitellogenin generate observed rank-specific phenotypes in other social insects (Hartfelder

320 2000), and dopamine may also play a central role in the regulation of dominance and

321 reproduction in species with simple societies (Sasaki et al. 2007; Okada et al. 2015; Ohkawara

322 and Aonuma 2016). Further work combining behavioural, genetic and physiological work is

323 needed to illuminate the regulatory underpinning of reproductive hierarchies in simple ant

324 societies. When looking at the broader phylogenetic picture there is accumulating evidence that

$325 \mathrm{JH}$ occupies a stunning range of different, often opposite, regulatory functions. The question of

326 how such incredible regulatory flexibility is possible without compromising fitness-relevant

327 functions is intriguing and a promising target for further molecular and comparative

328 investigations.

\section{REFERENCES}

332

333

334

335

336

Anderson, M., Gorley, R., Clarke, K. (2008) PERMANOVA+ for PRIMER: Guide to Software and Statistical Methods.

Asher, C. L., Nascimento, F. S. F., Sumner, S., Hughes, W. O. H. (2013) Division of labour and risk taking in the dinosaur ant, Dinoponera quadriceps (Hymenoptera: Formicidae). Myrmecological News. 18: 121-129. 
337 Azevedo, D. O., de Paula, S. O., Zanuncio, J. C., Martinez, L. C., Serrão, J. E. (2016) Juvenile

338 hormone downregulates vitellogenin production in Ectatomma tuberculatum (Hymenoptera:

339 Formicidae) sterile workers. J. Exp. Biol. 219: 103-108.

340 Bloch, G., Shpigler, H., Wheeler, D., Robinson, G. (2009) Endocrine influences on the

$341 \quad$ organization of insect societies. Hormones. 2: 1027-1068.

342 Bourke, A. F., Green, H. A., Bruford, M. W. (1997) Parentage, reproductive skew and queen 343 turnover in a multiple-queen ant analysed with microsatellites. Proc. R. Soc. B. 264: 277344283.

345 Brent, C., Peeters, C., Dietemann, V., Crewe, R., Vargo, E. (2006) Hormonal correlates of 346 reproductive status in the queenless ponerine ant, Streblognathus peetersi. J. Comp. Physiol. 347 A. 192: 339-339.

348 Brent, C. S., Penick, C. A., Trobaugh, B., Moore, D., Liebig, J. (2016) Induction of a 349 reproductive-specific cuticular hydrocarbon profile by a juvenile hormone analog in the 350 termite Zootermopsis nevadensis. Chemoecology. 26: 195-203.

351

352

353 354 355 356 357 358 359

Brent, C. S., Vargo, E. L. (2003) Changes in juvenile hormone biosynthetic rate and whole body content in maturing virgin queens of Solenopsis invicta. J. Insect Physiol. 49: 967-974.

Cant, M. A., Llop, J. B., Field, J. (2006) Individual variation in social aggression and the probability of inheritance: theory and a field test. Am. Nat. 167: 837-852.

Cuvillier-Hot, V., Lenoir, A., Crewe, R., Malosse, C., Peeters, C. (2004a) Fertility signalling and reproductive skew in queenless ants. Anim. Behav. 68: 1209-1219.

Cuvillier-Hot, V., Lenoir, A., Peeters, C. (2004b) Reproductive monopoly enforced by sterile police workers in a queenless ant. Behav. Ecol. 15: 970-975.

Dolezal, A. G., Brent, C. S., Gadau, J., Hölldobler, B., Amdam, G. V. (2009) Endocrine 
physiology of the division of labour in Pogonomyrmex californicus founding queens. Anim. Behav. 77: 1005-1010.

362

363

364

365

366

367

368

369

370

371

372

373

374

375

376

377

378

379

380

381

382

Field, J., Solis, C., Queller, D., Strassmann, J. (1998) Social and genetic structure of paper wasp cofoundress associations: tests of reproductive skew models.

Gotelli, N. J., Ellison, A. M. (2004) A Primer of Ecological Statistics. Sinauer Associates Publishers, Massachusetts, USA

Grainger, D. P., Asher, C. L., Jones, J. C., Nascimento, F. S., Sumner, S., Hughes, W. O. H. (2014) A novel method of assessing dominance hierarchies shows nuance, linearity and stability in the dinosaur ant Dinoponera quadriceps. Ethology. 120: 1073-1080.

Hartfelder, K. (2000) Insect juvenile hormone: From "status quo" to high society. Brazilian J. Med. Biol. Res. 33: 157-177.

Hölldobler, B., Wilson, E. O. (1990) The ants. Harvard University Press, Berlin

Jamieson, I. G. (1997) Testing reproductive skew models in a communally breeding bird, the pukeko, Porphyrio porphyrio. Proc. R. Soc. B. 264: 335-340.

Johnstone, R. (2000) Models of reproductive skew: A review and synthesis (invited Article). Ethology. 106: 5-26.

Kelstrup, H. C., Hartfelder, K., Nascimento, F. S., Riddiford, L. M. (2014) The role of juvenile hormone in dominance behavior, reproduction and cuticular pheromone signaling in the caste-flexible epiponine wasp, Synoeca surinama. Front. Zool. 11: 78-103.

Kelstrup, H. C., Hartfelder, K., Wossler, T. C. (2015) Polistes smithii vs. Polistes dominula: the contrasting endocrinology and epicuticular signaling of sympatric paper wasps in the field. Behav. Ecol. Sociobiol. 69: 2043-2058.

Kokko, H. (2003) Are reproductive skew models evolutionarily stable? Proc. R. Soc. B. 270: 
384 Kokko, H., Johnstone, R. a. (1999) Social queuing in animal societies: a dynamic model of 385 reproductive skew. Proc. R. Soc. B. 266: 571-578.

386

387

Libbrecht, R., Corona, M., Wende, F., Azevedo, D. O., Serrao, J. E., Keller, L. (2013) Interplay between insulin signaling, juvenile hormone, and vitellogenin regulates maternal effects on polyphenism in ants. Proc. Natl. Acad. Sci. U. S. A. 110: 11050-11055.

Monnin, T., Peeters, C. (1998) Monogyny and regulation of worker mating in the queenless ant Dinoponera quadriceps. Anim. Behav. 55: 299-306.

Monnin, T., Peeters, C. (1999) Dominance hierarchy and reproductive conflicts among subordinates in a monogynous queenless ant. Behav. Ecol. 10: 323-332.

Monnin, T., Ratnieks, F. L. W., Brandão, C. R. F. (2003) Reproductive conflict in animal societies: hierarchy length increases with colony size in queenless ponerine ants. Behav. Ecol. Sociobiol. 54: 71-79.

Nascimento, F. S., Souza, D. I. S. A., Tannure-Nascimento, I. C., Dantas, J. O. (2012) Social facilitation and food partitioning in the queenless ant Dinoponera quadriceps (Hymenoptera : Formicidae). J. Nat. Hist. 46: 1959-1967.

Neff, B., Pitcher, T., Ramnarine, I. (2008) Interpopulation variation in multiple paternity and reproductive skew in the guppy. Mol. Ecol. 17: 2975-2984.

Nijhout, H. F. (1998) Insect Hormones. Princeton University Press, Princeton

Norman, V. C., Hughes, W. O. H. (2016) Behavioural effects of juvenile hormone and their influence on division of labour in leaf-cutting ant societies. J. Exp. Biol. 219: 8-11.

Ohkawara, K., Aonuma, H. (2016) Changes in the levels of biogenic amines associated with aggressive behavior of queen in the social parasite ant Vollenhovia nipponica. Insectes Soc. 
63: 257-264.

407 Okada, Y., Sasaki, K., Miyazaki, S., Shimoji, H., Tsuji, K., Miura, T. (2015) Social dominance

408 and reproductive differentiation mediated by dopaminergic signaling in a queenless ant. J.

$409 \quad$ Exp. Biol. 218: 1091-1098.

410

411

412

413

414

415

416

417

418

419

420

421

422

423

424

425

426

427

428

Pamminger, T., Buttstedt, A., Norman, V., Schierhorn, A., Botías, C., Jones, J. C., Basley, K., Hughes, W. O. H. (2016a) The effects of juvenile hormone on Lasius niger reproduction. J. Insect Physiol. 95: 1-7.

Pamminger, T., Treanor, D., Hughes, W. O. H. (2016b) Pleiotropic effects of juvenile hormone in ant queens and the escape from the reproduction-immunocompetence trade-off. Proc. Biol. Sci. 283: 20152409-.

Patalano, S., Vlasova, A., Wyatt, C., Ewels, P., Camara, F., Ferreira, P. G., Asher, C. L., Jurkowski, T. P., Segonds-Pichon, A., Bachman, M., González-Navarrete, I., Minoche, A. E., Krueger, F., Lowy, E., Marcet-Houben, M., Rodriguez-Ales, J. L., Nascimento, F. S., Balasubramanian, S., Gabaldon, T., Tarver, J. E., Andrews, S., Himmelbauer, H., Hughes, W. O. H., Guigó, R., Reik, W., Sumner, S. (2015) Molecular signatures of plastic phenotypes in two eusocial insect species with simple societies. Proc. Natl. Acad. Sci. U. S.

A. 112: 13970-13975.

Pearce, A. N., Huang, Z. Y., Breed, M. D. (2001) Juvenile hormone and aggression in honey bees. J. Insect Physiol. 47: 1243-1247.

Peeters, C. (1997) Morphologically “primitive” ants: comparative review of social characters, and the importance of queen-worker dimorphism. In: Choe JC, Crespi BJ (eds) The evolution of social behavior in insects and arachnids. pp 372-391

Penick, C. A., Liebig, J. (2017) A larval 'princess pheromone' identifies future ant queens based 
on their juvenile hormone content. Anim. Behav. 128: 33-40.

430 Penick, C. A., Liebig, J., Brent, C. S. (2011) Reproduction, dominance, and caste: endocrine

431 profiles of queens and workers of the ant Harpegnathos saltator. J. Comp. Physiol. A. 197:

$432 \quad 1063-1071$.

433 Ratnieks, F. L. W., Foster, K. R., Wenseleers, T. (2006) Conflict resolution in insect societies. $434 \quad$ Annu. Rev. Entomol. 51: 581-608.

435 Ratnieks, F. L. W., Reeve, H. K. (1992) Conflict in single-queen hymenopteran societies: the 436 structure of conflict and processes that reduce conflict in advanced eusocial species. J. 437 Theor. Biol. 158: 33-65.

438

439

440

441

442

443

444

445

446

447

448

449

450

451

Reeve, K., Keller, L. (2001) Tests of reproductive-skew models in social insects. Annu. Rev. Entomol. 46: 347-85.

Robinson, G. E., Vargo, E. L. (1997) Juvenile hormone in adult eusocial hymenoptera: gonadotropin and behavioral pacemaker. Arch. Insect Biochem. Physiol. 35: 559-583.

Sasaki, K., Yamasaki, K., Nagao, T. (2007) Neuro-endocrine correlates of ovarian development and egg-laying behaviors in the primitively eusocial wasp (Polistes chinensis). J. Insect Physiol. 53: 940-949.

Smith, C. R., Suarez, A. V, Tsutsui, N. D., Wittman, S. E., Edmonds, B., Freauff, A., Tillberg, C. V (2011) Nutritional asymmetries are related to division of labor in a queenless ant. PLoS One. 6: e24011.

Sommer, K., Hölldobler, B., Rembold, H. (1993) Behavioral and Physiological Aspects of Reproductive Control in a Diacamma Species from Malaysia (Formicidae, Ponerinae). Ethology. 94: 162-170.

Tibbetts, E. A., Huang, Z. Y. (2010) The challenge hypothesis in an insect: juvenile hormone 
452 increases during reproductive conflict following queen loss in Polistes wasps. Am. Nat. $453 \quad 176: 123-130$.

454 Tibbetts, E. A., Izzo, A. S. (2009) Endocrine mediated phenotypic plasticity: condition455 dependent effects of juvenile hormone on dominance and fertility of wasp queens. Horm. $456 \quad$ Behav. 56: 527-531.

457 Tibbetts, E. A., Vernier, C., Jinn, J. (2013) Juvenile hormone influences precontest assessment 458 behaviour in Polistes dominulus paper wasps. Anim. Behav. 85: 1177-1181.

459 Tranter, C., LeFevre, L., Evison, S. E. F., Hughes, W. O. H. (2014) Threat detection: contextual 460 recognition and response to parasites by ants. Behav. Ecol. 396-405.

461 Widdig, A., Bercovitch, F. B., Streich, W. J., Sauermann, U., Nürnberg, P., Krawczak, M. (2004)

462 A longitudinal analysis of reproductive skew in male rhesus macaques. Proc. Biol. Sci. 271: $463 \quad 819-826$. 


\section{Figure legends}

467 Figure 1.Boxplots showing fertility estimators measured in 32 Dinoponera quadriceps high

468 ranked workers. Half of the ants were treated with Juvenile Hormone analogue (Methoprene) and

469 half with acetone control (CoA). Fertility measures were the average ovariole width (A),

470 maximum ovariole width (B), number of oocytes (C) and minimum ovariole width (D). Stars

471 above plots indicate significant differences following Wilcoxin tests.

472

473 Figure 2. Multidimensional scaling (MDS) plot of all behaviours measured in 32 Dinoponera

474 quadriceps high-ranked workers. Half of the ants were treated with Juvenile Hormone analogue

475 (JHa; red triangles) and half with acetone control (CoA; blue circles). Behaviours measured were

476 brood care, sociability as distance from nearest ant and number of ant contacts, activity,

477 'boldness' and aggression, as well as the change in rank following treatment. Vector lines

478 indicate the strength and contribution of the individual traits for group separation between the

479 two treatment groups. There were no significant differences between the treatments. 


\section{Figure 1}

Figure 1. Boxplots showing fertility estimators measured in 32 Dinoponera quadriceps high ranked workers

Half of the ants were treated with Juvenile Hormone analogue (Methoprene) and half with acetone control (CoA). Fertility measures were the average ovariole width (A), maximum ovariole width (B), number of oocytes (C) and minimum ovariole width (D). Stars above plots indicate significant differences following Wilcoxin tests. 

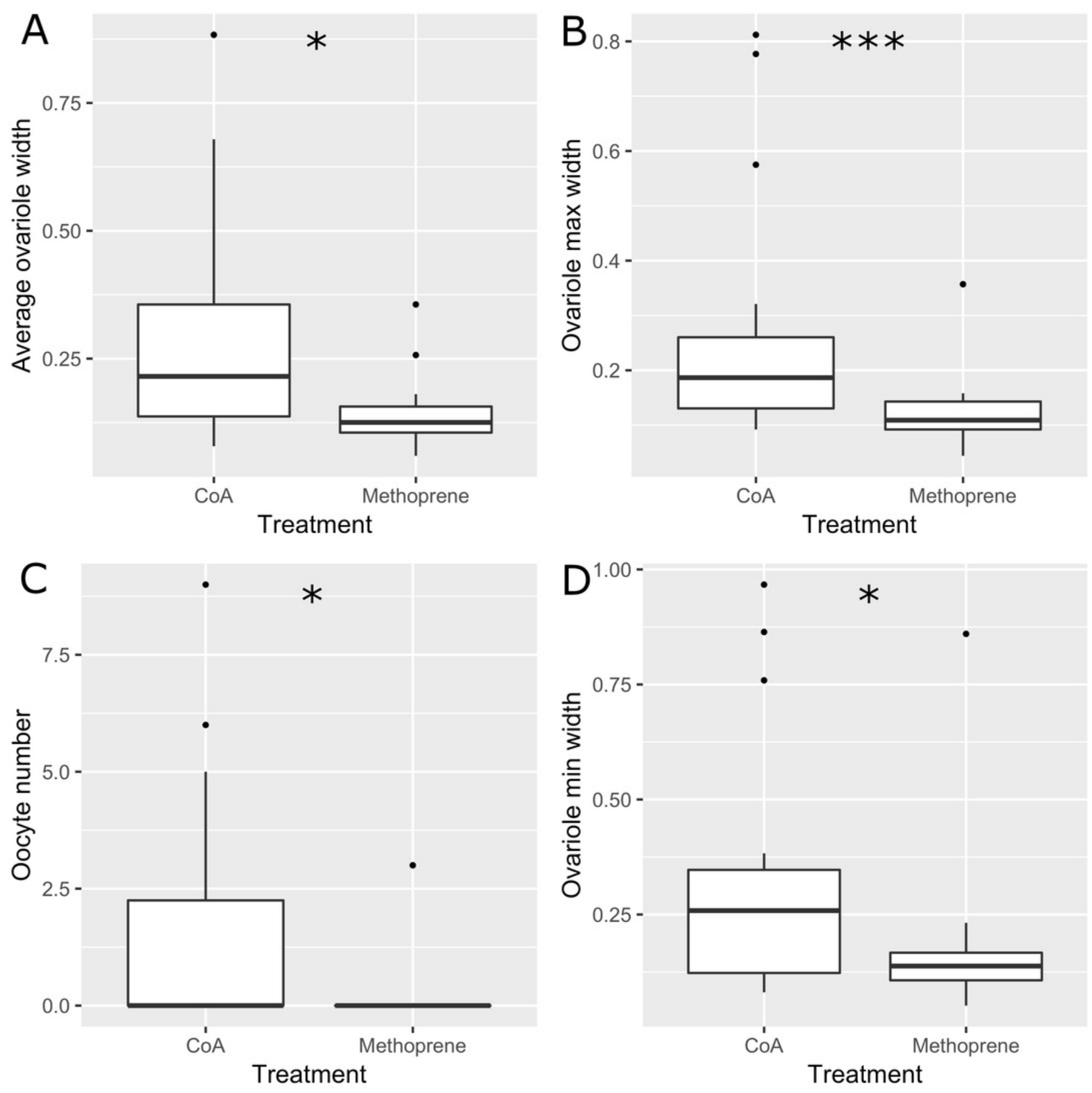


\section{Figure 2}

Figure 2. Multidimensional scaling (MDS) plot of all behaviours measured in 32 Dinoponera quadriceps high-ranked workers.

Half of the ants were treated with Juvenile Hormone analogue (JHa; red triangles) and half with acetone control (CoA; blue circles). Behaviours measured were brood care, sociability as distance from nearest ant and number of ant contacts, activity, 'boldness' and aggression, as well as the change in rank following treatment. Vector lines indicate the strength and contribution of the individual traits for group separation between the two treatment groups. There were no significant differences between the treatments.

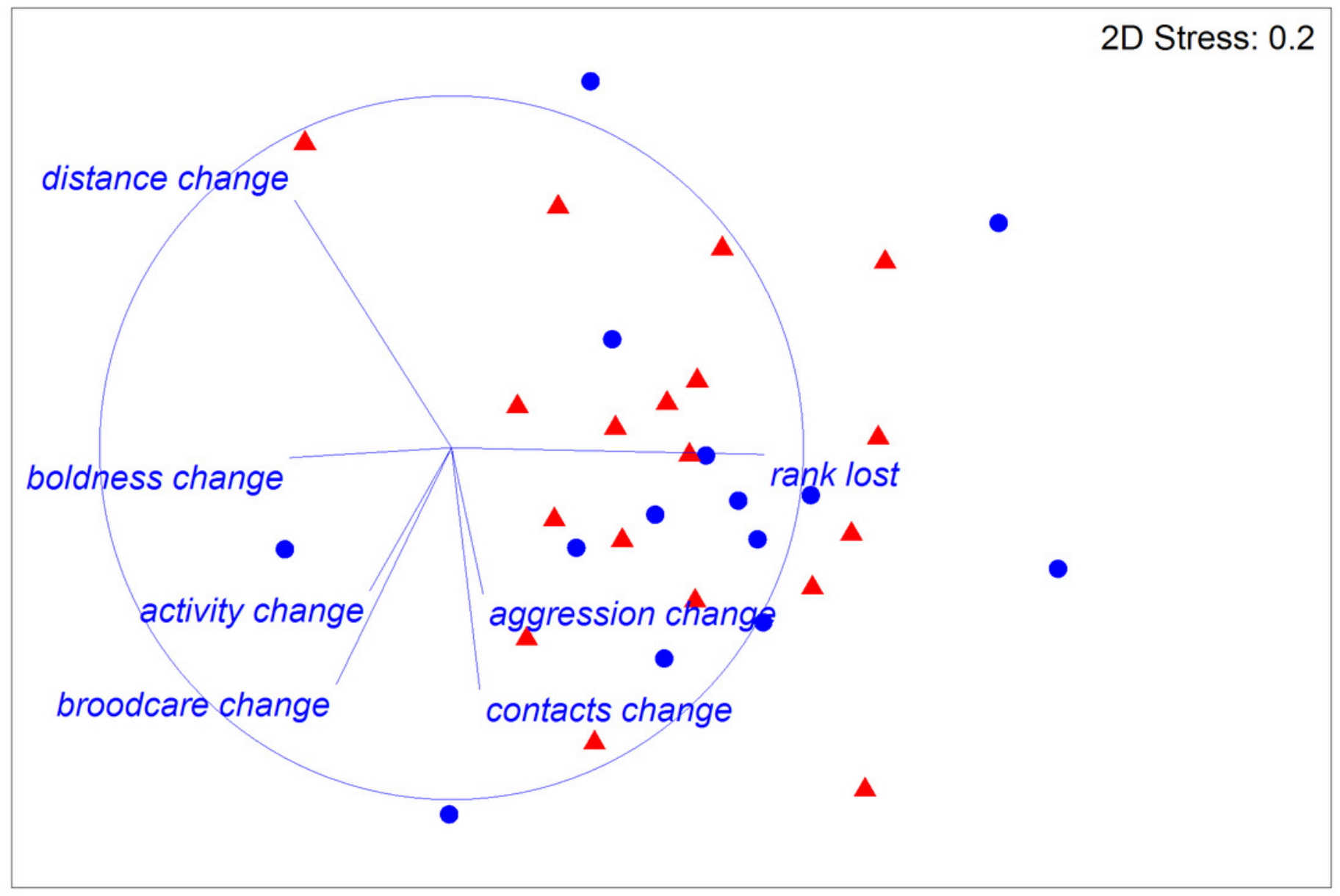




\section{Table $\mathbf{1}$ (on next page)}

Table 1. Results of the fertility SIMPER analysis.

Presented are the average distance between samples both within and between treatments, and the percentage contribution of the individual factors. 
1 Table 1: Median values for physiological traits between acetone solvent control (CoA)

2 individuals and methoprene treated Dinosaur ant indivduals

\begin{tabular}{|c|c|c|c|c|c|c|}
\hline & 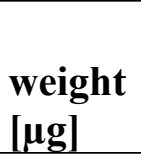 & $\begin{array}{l}\text { head } \\
\text { width } \\
\text { [mm] } \\
\end{array}$ & $\begin{array}{l}\text { Ovariole } \\
\text { max width }\end{array}$ & $\begin{array}{l}\text { Ovariole } \\
\text { min width }\end{array}$ & $\begin{array}{l}\text { Oovariole } \\
\text { average } \\
\text { width }\end{array}$ & $\begin{array}{l}\text { Oocyte } \\
\text { number }\end{array}$ \\
\hline CoA & 335 & 4.9 & 0.186 & 0.247 & 0.208 & 0 \\
\hline Methoprene & 374 & 4.959 & 0.1075 & 0.142 & 0.122333334 & 0 \\
\hline
\end{tabular}

3

4

5 


\section{Table 2 (on next page)}

Table 2. Results of the fertility Wilcoxin analyses comparing Dinosaur ant individuals treated with either acetone solvent control or methoprene.

Presented are the $\mathrm{W}$ test statistic and $\mathrm{P}$ values for each test carried out. 
1 Table 2. Results of the fertility Wilcoxin analyses comparing Dinosaur ant individuals treated

2 with either acetone solvent control or methoprene. Presented are the $\mathrm{W}$ test statistic and $\mathrm{P}$ values

3 for each test carried out.

\begin{tabular}{|l|c|c|}
\hline & W & P-value \\
\hline & & \\
\hline Oocyte number & 187.5 & 0.015 \\
\hline Ovariole width (minimum) & 200 & 0.021 \\
\hline Ovariole width (maximum) & 212.5 & 0.006 \\
\hline Ovariole width (average) & 202.5 & 0.017 \\
\hline
\end{tabular}

4

5 